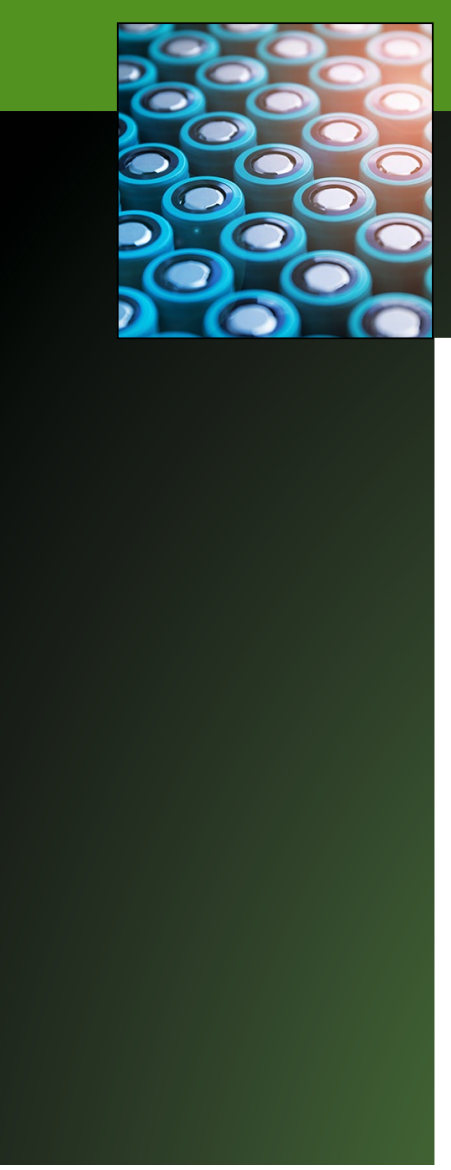

\title{
New developments in battery safety for large-scale systems
}

\author{
Joshua Lamb* and Judith A. Jeevarajan, Guest Editors
}

\begin{abstract}
Battery safety is a multidisciplinary field that involves addressing challenges at the individual component level, cell level, as well as the system level. These concerns are magnified when addressing large, high-energy battery systems for grid-scale, electric vehicle, and aviation applications. This article seeks to introduce common concepts in battery safety as well as common technical concerns in the safety of large rechargeable systems. Lithium-ion batteries represent the most significant technology in high-energy rechargeable batteries and a technology with well-known safety concerns. Because of this, particular attention is paid to introduce common concepts and concerns specific to these batteries. An introduction of system-level battery issues that may cause problems in larger systems is given. Finally, a brief summary of the gaps in emergent technologies is provided. As most of the effort in new technologies goes toward improving performance, there are significant gaps in understanding safety performance of these new batteries.
\end{abstract}

\section{Introduction}

Battery safety is a difficult concept to quantify. For a typical end user, safety is often a binary quality; either their battery has operated without incident, or it did not. Generally, users do not want to consider the battery at all in general operation; the best battery is the one that simply works without giving it much thought. Ultimately, when discussing safety, we are really discussing the level of risk a user is willing to accept for a given application, and the potential consequences of a failure. This requires methods to evaluate both the severity of potential events and the probability they will occur. Risk assessment evaluations are typically conducted following matrixes such as those seen in Figure 1. The challenge with battery systems is that while severity can often be evaluated through laboratory testing, the probability of failure can be difficult to determine during the early adoption phase of a new technology. This issue will discuss common issues and solutions for understanding and improving the safety performance of battery technologies, particularly those of interest to stationary, electric vehicle, and aviation applications.

The idea of battery safety is often inseparable from battery fires and thermal runaway events. Thermal runaway is ultimately an inherent risk of the stored energy. In the case of batteries, thermal runaway occurs when an external or internal condition, causes the energy stored within the battery to begin to be released at a rate faster than it can be dissipated resulting in an uncontrolled temperature rise of the battery than can then result in violent venting, fire, and smoke. If the natural or forced cooling of the battery is sufficient to remove this excess heat, the event can be stopped before thermal runaway occurs.

The most common means of understanding battery safety is through battery abuse testing. Abuse testing refers to tests that are off-nominal and abusive in nature and the tests typically replicate credible but off-nominal conditions that can be encountered if the battery is not designed or used correctly. Abuse tests help to identify the safety limits of the battery and design the relevant controls and also provide information on the worst case results in the event such an off-nominal condition is levied on the battery. Abuse testing manuals and standard testing procedures are available from several organizations, with examples in References 1-5. This effectively determines the "severity" axis of Figure 1 by applying various extreme conditions to the battery to determine the worst-case scenarios for a particular battery. The space industry has been able to come up with an approach to test cells and batteries to credible off-nominal conditions for human-rated missions. ${ }^{6}$ The referenced article $^{6}$ also includes the approach that the US Navy took during that time period of the article publication. It is imperative that a thorough analysis be performed on the batteries 


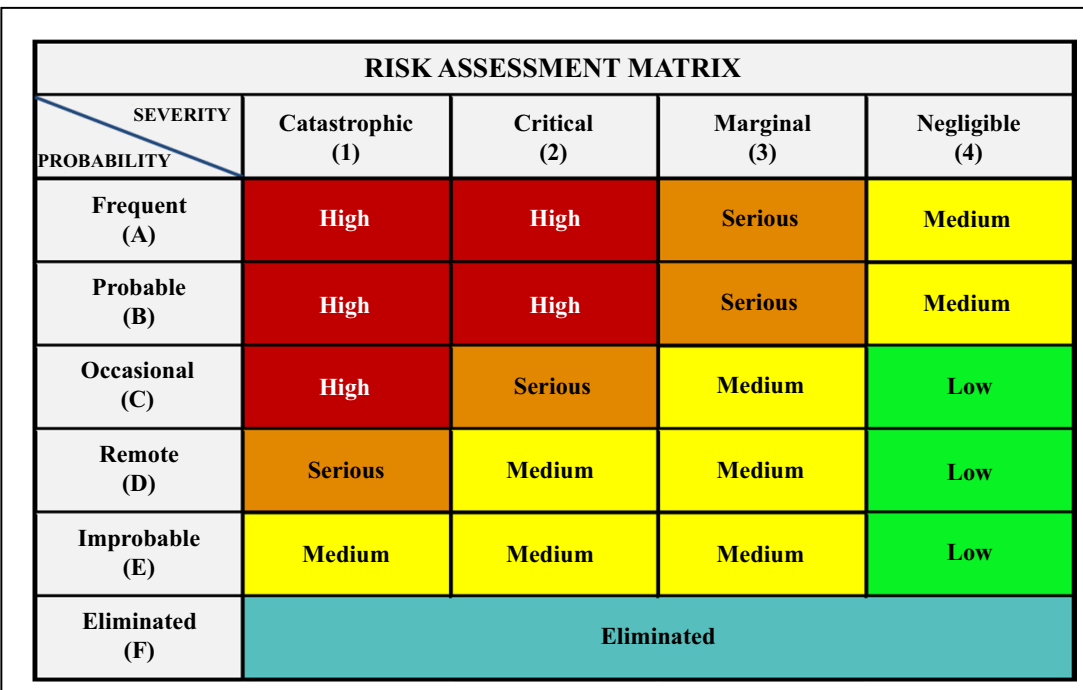

Figure 1. A risk assessment matrix adapted from MIL-STD-882E, Department of Defense Standard Practice: System Safety.

(NCA). These materials offer high potential energy storage, but are also unstable under a wide variety of conditions. These are most susceptible to the classic thermal runaway scenario. When exploring cathode materials beyond layered metal oxides, similar behaviors are often observed, but the mechanisms may change and the temperatures of various events can vary significantly. Cathode materials such as $\mathrm{LiFePO}_{4}$ (LFP) and $\mathrm{LiMn}_{2} \mathrm{O}_{4}$ (LMO), in particular, do not typically exhibit energetic cathode breakdown, but will still exhibit electrolyte breakdown as well as SEI and anode decomposition. Further, the decomposition products from common electrolytes are typically flammable and present a source of energy during thermal

in the environment they are used in and the tests should include the relevant configuration. The designer and user of any large energy-storage system must be aware of the risks they are accepting, and although a significant challenge in terms of cost and time, carry out all relevant abuse testing to fully understand the consequences of those credible offnominal conditions as this will provide adequate information to design and deploy the system with a reduced level of risk.

\section{Safety of lithium-ion cells and batteries}

Lithium-ion cell chemistry has the highest energy density of all battery chemistries available in the market today that also provides a significantly high cycle and calendar life as well as a range of rate capabilities. However, associated with these advantages is the propensity of cells and batteries of this chemistry to undergo catastrophic failures if not designed or used in the relevant and appropriate manner. This leads one to conclude that if some of the stringent procedures of manufacturing and design control are exercised, one can reduce the risk posed by $\mathrm{Li}$-ion. For instance, if the quality and manufacturing control are followed stringently, if the cells and batteries are used well within the manufacturer's specification, if the battery safety controls are set stringently and appropriately, and if the relevant charger is used, then the risk of failure can be minimized. Some of these factors will be discussed next in more detail.

\section{Battery chemistry}

The specific chemistry of lithium-ion batteries is often seen as a major factor in the safety performance of li-ion. The most commonly used cathode materials are the layered metal oxides $\mathrm{LiCoO}_{2}(\mathrm{LCO}), \mathrm{LiNi}_{\mathrm{x}} \mathrm{Mn}_{\mathrm{y}} \mathrm{Co}_{\mathrm{z}}(\mathrm{NMC})$, and $\mathrm{LiNi}_{\mathrm{x}} \mathrm{Co}_{\mathrm{y}} \mathrm{Al}_{\mathrm{z}}$ runaway unrelated to the active materials. ${ }^{7}$ Sandia National Laboratories has previously used accelerating rate calorimetry (ARC) as a basis to compare these materials with an example of this shown in Figure 2. This shows thermal runaway events of various battery technologies compared in terms of their observed heating rate as a function of temperature. While there is a plethora of information available here, reducing the severity of thermal runaway events means reducing both the width and height of observed peaks. Noticeable on this scale is that the LFP peak here is basically flat compared to other chemistries, with orders of magnitude less energy release than the layered metal oxide cathodes. However, this comes at a cost of reduced specific energy and energy densities, impacting the potential energy-storage performance if chosen for an application.

\section{Quality and manufacturing control}

Lithium-ion cells require clean room and dry room environments to manufacture high-quality cells. Lowering the quality of the lithium-ion cells not only lowers its performance with respect to capacity and internal resistance, but also its cycle and calendar life. In addition to this, lowering the quality of the manufacturing process can adversely affect the safety of these cells. Lack of stringent environmental conditions during the manufacturing process can introduce defects such as foreign object or native object debris, lack of alignment of the electrodes and separator, tears and holes in the electrodes and separator, bad welds that can damage the separator, and lack of insulation in metallic parts such as tabs used in the construction of the cell components. Some of these defects may not be detected during the manufacturing process and may manifest themselves as failures in the field. Hence, high-quality 

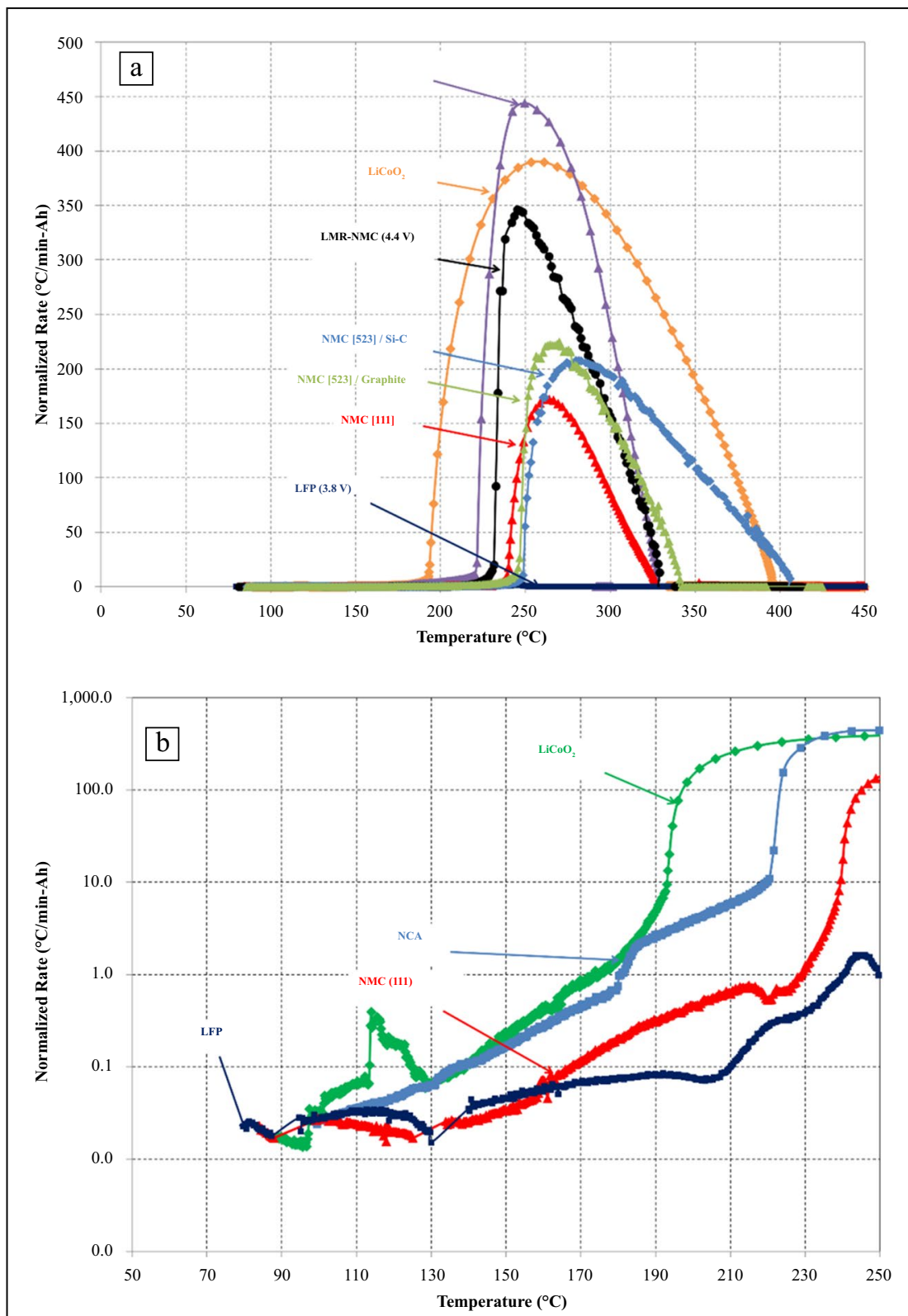

Figure 2. Accelerating rate calorimetry showing thermal runaway events of various active materials. (a) The peak width of these plots can generally be interpreted as the total energy released during thermal runaway with the peak height relating to how rapidly heating is able to occur. Other important values include the onset temperature of the peak, showing the temperature at which high rate thermal runaway will occur. (b) Select plots are also shown in a log scale showing the lower temperature, low rate behavior comparison of these cathode materials.

manufacturing processes along with stringent quality control is imperative to reduce the risk of failures during the life of the cell or battery.

\section{Usage of cells and batteries within the manufacturer's specification}

Lithium-ion cells and batteries should stringently follow manufacturer's specifications for current, voltage and temperature to reduce the risk of failures. As one exceeds the manufacturing specification for voltage, overcharge or overdischarge can occur. Overcharge results in the formation of lithium dendrites, decomposition of electrolyte and destabilization of cathode leading to easy release of oxygen from the cathode. And during an overcharge, the temperature of the cell goes up and this effect along with the other reactions just mentioned leads to a fire and thermal runaway event. Although overdischarge in itself does not result in a catastrophic hazard, it does cause electrolyte decomposition with gas production and dissolution of copper. Extreme overdischarge causes the cell to become a dead cell. Subtle overdischarges followed by charge causes the dissolved copper to deposit as copper metal over the cathode, anode and separator. This can then hinder the intercalation of lithium ions in the anode during the charge process causing heat to be produced that can then result in thermal runaway. ${ }^{8-11}$

In general, a reduced voltage range will help to avoid the side reactions that occur at the two ends of the voltage range and provides a higher margin for safety. Studies have also shown that reducing the voltage range can more than double the number of cycles obtained with Li-ion. ${ }^{1-13}$ Traditionally, highquality portable electronic equipment are designed with appropriate protocols for charge and discharge. ${ }^{14}$ The charger reduces the charge current before the end of charge voltage is reached and there are controls on the battery as well as the device that controls the voltage that the battery is allowed to discharge to. In addition, the battery management system (BMS) installed internal to the battery design monitors and controls the voltage of every cell bank, the battery level current and in many cases the temperature. Some BMSs also perform the function of balancing the cells/ cell banks in a string. 


\section{System-level battery management}

All lithium-ion batteries, irrespective of number of cells, voltage and capacity, will typically feature some level of control circuitry. Battery management systems (BMS), even on larger devices, can vary significantly in the level of rigor. All rechargeable battery systems feature some level of control and monitoring. Lithium-ion batteries are particularly sensitive to out of specification voltage and temperature, and as such, are typically installed with a more complex BMS. This is particularly true of complex batteries featuring several cells in both series and parallel configurations, as maintaining voltage balance and appropriate temperature of the cells is critical for safe operation. The greater the hazard, the more complex the BMS becomes, with resulting penalties to specific energy and energy density.

The BMS can provide protection against overvoltage, undervoltage, and external short circuits. There is currently, however, no widely accepted practice for the detection of an internal short circuit. Internal short circuit is a spontaneous failure of a single cell driven by the development of a short circuit between the cathode and anode electrodes. Internal shorts can occur in two ways. The first is due to a manufacturing defect that was undetected in the manufacturing facility and results in a failure in the field. The second occurs when internal shorts are created due to an off-nominal condition such as overcharge where lithium dendrites are formed, overdischarge where copper dissolution and copper metal deposition occurs and external shorts where an intense hot spot is created internal to the cell melting the separator causing the shorts. Internal shorts can occur due to manufacturing defects such as foreign object debris, tears in the separator, metal filings embedded in the electrodes, lack of insulation tape over the metal tabs, misalignment of the electrode wind, compression of the edges of the electrode wind, etc. Although this type of internal short occurs with relatively low frequency, if the single cell failure propagates, it could result in a catastrophic thermal runaway of the entire battery. The second type of internal short that occurs due to improper controls can be avoided by designing the controls correctly. The health monitoring of fielded batteries typically focuses on voltage, current and sometimes temperature monitoring of the pack. This is particularly difficult with large packs that feature multiple cells in parallel, as the cells in parallel will naturally balance their voltage, making deviations in voltage due to an internal short circuit virtually impossible. Since this can lead to a thermal runaway of a single cell, this raises concerns in an application where the consequence of failure is high, such as in aviation applications. Without the ability to adequately detect and arrest these failures, the design of propagation resistant battery packs is the current solution. This however, comes at a significant cost in system-level energy density.

\section{Propagation resistance}

The design of packs of both high energy density and high reliability presents a specific challenge in regards to preventing a single-cell thermal runaway from propagating to neighboring cells. Also, most consumer-level safety devices are designed with low voltage consumer electronics in mind and are often inadequate or become the cause of failure in high-energy battery packs. ${ }^{14}$ The principal concern is preventing a single cell failure from propagating to the surrounding pack. Randomized single cell failures, while rare, are difficult to fully prevent. When a system-level failure is an unacceptable consequence, the primary solution is ultimately designing the system to be robust to failure propagation. Propagation resistance is typically tested by initiating a single cell within a battery and observing if the thermal runaway of the single cell propagates to neighboring cells and further through the pack. Various testing organizations have developed procedures for performing this test. ${ }^{15-18}$

Current strategies use a combination of cell selection, spacing of cells, ${ }^{19}$ and interstitial material to build passive propagation resistance into the pack. Various organizations are currently working on understanding the heat dissipation and conduction properties in the batteries to design safer batteries with thermal runaway propagation resistance characteristics.

While vehicle original equipment manufacturers (OEMs) typically do not have the same reliability requirements seen in Space and Defense missions, they have fielded the largest numbers of high capacity and high energy density systems to date, and in some cases, have developed some strategies toward failure propagation mitigation.

Propagation-resistant designs typically focus on cylindrical cells such as those previously patented by Tesla, as well as those used in batteries designed for NASA space applications. ${ }^{18-21}$ Jeevarajan' $\mathrm{s}^{21}$ work also shows the limitations of cell-level safety devices, as devices that are used to prevent cell-level failure such as positive temperature coefficient (PTC) and current interrupt devices (CID) are nonfunctional at high voltages or can even initiate fires in extreme cases. The geometric inefficiency of cylindrical cells also limits at least the volumetric energy density, as significant space is lost in the pack design. Pouch format cells offer a much higher energy density $(\mathrm{Wh} / \mathrm{L})$, and even a higher specific energy $(\mathrm{Wh} / \mathrm{kg})$ as they typically are enclosed in a lighter aluminized polymer wrapping. The design of terminal tabs in pouch cells which are relatively fragile make spacing between them more difficult to maintain and the pouch cells burst open at pressures as low as 50 psi releasing flammable liquid electrolyte and vapors resulting in a larger fire. Hence, these are significantly more challenging to design in a system that takes propagation resistance into account.

\section{Emergent technologies}

Driven by electrification of transportation and grid sectors, industries and governments globally have funded the development of next-generation high energy density rechargeable cells that are either based on silicon-anode or Li-metal anode. 
Li-ion based on $\mathrm{Si}$ anode and Li metal rechargeable cells have demonstrated specific energy $\sim 400 \mathrm{Wh} / \mathrm{kg}$ at $5 \mathrm{~h}$ or longer discharge rates. Safety studies on these materials are not widely available, however, as safety testing typically requires cells of at least 1-5 amp hours in size. This limits the testing of materials developed at the laboratory scale. While some manufacturers have begun producing larger format silicon anode cells most are reluctant to publish safety testing data on early stage product development in a publicly available forum.

Much of the focus of battery safety research has been on lithium-ion batteries due to both their broad applicability in rechargeable energy-storage systems, as well as well documented safety vulnerabilities discussed throughout this issue. Stationary energy-storage programs, in particular, do not have the same specific energy and energy density requirements, but do require batteries to be able to provide high reliability for a long effective life to be viable. Alternative technologies include molten salt chemistries, advanced rechargeable alkaline chemistries, and flow battery chemistries. While many of these technologies are assumed to carry less risk than the prevalent lithium-ion technologies, they are also significantly less well studied. Recent work by Wittman et al. ${ }^{22}$ provided a perspective on the state of safety research for aqueous battery technologies and demonstrated that there are known areas of concern that have been poorly studied in large battery systems. An overview of the general concerns in aqueous and other non-lithium systems is provided as well by Koehler. ${ }^{23}$ This provides discussion on concerns around high-temperature battery systems as well, which are of interest in large stationary storage systems.

System-level improvements are an attractive target for improving energy density of batteries while still maintaining high reliability. The notion is that improving safety at the system level would allow the use of high energy density cell chemistries while still maintaining high reliability. A strategy currently being pursued is advanced methods for failure detection. This is of interest for improving the safety of lithiumion systems. The goal is to provide a means for detecting single-cell failure during early stages before a catastrophic thermal runaway. With demonstrated reliability, these strategies could be an alternative to propagation resistance. Rather than ensuring a thermal runaway is unable to propagate, the system would detect the onset of failure early and allow for intervention. There is current research and development in the area of early detection, suggesting a breakthrough is possible in the near term. CAMX Power has developed and marketed a device claimed to detect internal short circuits. ${ }^{24}$ While little details on the operating principals of the technique are publicly available due to intellectual property (IP) considerations, public demonstrations have been promising and the device is a size that is suitable for on-board integration.

Electrochemical impedance spectroscopy (EIS) is a potentially powerful method for interrogating batteries, as it can provide a snapshot of the electrochemical state of a battery, however, the equipment is typically limited to the laboratory due to the time and expertise required for making measurements. An overview of applying traditional EIS techniques to commercial Li-ion cells is provided by Meddints et al. ${ }^{25}$ New techniques and hardware strategies are being explored to make it more useful as a diagnostic and monitoring tool. Love et al. ${ }^{26,27}$ as well Srinivasan et al. ${ }^{28-30}$ have explored using single-frequency EIS measurements to detect changes in battery temperatures as well to evaluate the health of a cell. Single-frequency measurements are comparatively fast and easier to evaluate making them ideal for on-board monitoring. Idaho National Laboratories, working with Montana Tech, ${ }^{31-33}$ developed a tool capable of making full spectrum measurements in a fraction of the normal time. This has been used at Sandia National Laboratories to evaluate lithium-ion cells under abuse conditions ${ }^{34}$ This hardware is currently at the laboratory benchtop scale and significant miniaturization is needed to make it appropriate for on-board monitoring.

Advanced materials to mitigate cell-to-cell failure propagation are also being explored. Phase-change materials have been proposed ${ }^{18,35}$ by various parties as a potential propagation barrier. The current technical challenge with phase-change materials is that thermal management is also needed for normal operation. Phase-change materials are typically tuned to be active at a specific temperature. Temperatures where thermal runaway is a concern are often much higher than the normal operating temperatures of a battery, so phase-change materials can often be tuned for normal thermal management, or thermal runaway mitigation, but not both.

\section{Summary}

All stored energy carries an inherent risk, and batteries are not immune from this. This is often forgotten, because the well-designed batteries out there simply work. They perform their jobs quietly and effectively, allowing the use of electronic devices that are increasingly hungry for power. The consumer electronics industry has seen a large number of recalls in the past two decades due to unforeseen challenges that always exist with new materials and designs. And when building large systems for grid-scale energy storage, vehicle electrification, or even electric powered flight we are often commissioning increasingly significant amounts of stored energy concentrated within a battery system. Large battery systems such as this are ultimately a relatively new technology without the benefit of the decades of experience with other more established energy storage technologies and fuels. This issue will explore active research and development activities to better understand, predict, and mitigate battery failure and drive toward safer energy-storage systems.

In summary, cells and batteries of the lithium-ion battery chemistry can cause catastrophic failures if not manufactured with a high quality or used within the manufacturer's specifications. This issue has a range of articles that discuss the properties that can affect safety and methods that can be used to mitigate the catastrophic failures. In this issue, authors will first describe the thermodynamic processes that lead to 
thermal runaway. An overview is provided of the reaction processes that lead to thermal runaway as well as tools that can be used to predict this behavior. These understandings can then be applied in multiscale approaches to better understand the risks posed by cells and battery systems. Hewson, Mukherjee et al. ${ }^{36}$ provide a detailed explanation of the material processes that can affect safety, which include the anode-electrolyte and cathode electrolyte interactions and use multiscale modeling to describe how material properties can be changed to improve the safety of systems of this chemistry.

Methods for evaluating the state of health and state of safety of batteries are also explored. Articles examine various approaches that can be used to understand the electrochemical behavior of batteries as they approach failure. Thermophysical analysis can also be used to better understand the material properties of cells as they approach failure as well as provide an alternate means to investigate cells during operation that is not reliant on or influenced by the active electrical connection to the battery. Steingart ${ }^{37}$ discusses the thermophysical properties and its relationship to the safety of the cells and includes methods to characterize and understand the worst-case catastrophic results at the cell level. Tanim et al. ${ }^{38}$ cover the areas of concerns when one goes from the cell level to a system level and the disadvantages of extending cell-level safety test data to safety at the system level. They also emphasize the need to test at the system level to get the comprehensive picture with respect to safety of the system.

Finally, in this issue we explore case studies in safety-critical operations, as well as potential methods for mitigating system-level failures. Takahashi ${ }^{39}$ provides an excellent coverage of the materials that can be used to mitigate or contain a fire and discusses some of the materials tested for their properties to provide such protection for lithium-ion systems. Viswanathan $^{40}$ discusses the use of high energy density cells necessary for electric powered flight that also provides a study of a safety-critical application.

\section{Acknowledgments}

Sandia National Laboratories (SNL) is a multimission laboratory managed and operated by National Technology \& Engineering Solutions of Sandia, LLC, a wholly owned subsidiary of Honeywell International Inc., for the US Department of Energy's National Nuclear Security Administration under Contract No. DE-NA0003525. This article describes objective technical results and analysis. Any subjective views or opinions that might be expressed in the paper do not necessarily represent the views of the US Department of Energy or the United States Government. Battery safety research at SNLs is supported through funding from the Department of Energy, Office of Electricity, the Department of Energy Vehicle Technologies Office, and the Department of Transportation
National Highway Traffic Safety Administration. We would like to thank I. Gyuk, D. Howell, S. Boyd, S. Gillard, and S. Summers for their continued support of battery safety research at SNL. Underwriters Laboratories (UL) is a nonprofit organization dedicated to advancing the UL public safety mission through the discovery and application of scientific knowledge. We conduct rigorous independent research and analyze safety data, convene experts worldwide to address risks, share knowledge through education and public outreach initiatives, and develop standards to guide safe commercialization of evolving technologies. We foster communities of safety, from grassroots initiatives for neighborhoods to summits of world leaders. Our organization employs collaborative and scientific approaches with partners and stakeholders to drive innovation and progress toward improving safety, security, and sustainability, ultimately enhancing societal well-being. Underwriters Laboratories has sponsored the Open Access of this issue, which is in line with their mission of making the world a safer place through the sharing of scientific knowledge.

\section{Conflict of interest}

Sandia National Laboratories is a multimission laboratory managed and operated by National Technology \& Engineering Solutions of Sandia, LLC, a wholly owned subsidiary of Honeywell International Inc., for the US Department of Energy's National Nuclear Security Administration under Contract No. DE-NA0003525. Funding for battery safety research has been provided by the US Department of Energy Office of Electricity, the US Department of Energy Vehicle Technologies Office, and the US Department of Transportation. The authors do not anticipate that any of these organizations stand to gain or lose financially from the publication of this work. Underwriters Laboratories is a nonprofit organization dedicated to advancing the UL public safety mission through the discovery and application of scientific knowledge. The authors do not anticipate that Underwriters Laboratories stands to gain or lose financially from the publication of this work.

\section{Open Access}

This article is licensed under a Creative Commons Attribution 4.0 International License, which permits use, sharing, adaptation, distribution and reproduction in any medium or format, as long as you give appropriate credit to the original author(s) and the source, provide a link to the Creative Commons licence, and indicate if changes were made. The images or other third party material in this article are included in the article's Creative Commons licence, unless indicated otherwise in a credit line to the material. If material is not included in the article's Creative Commons licence and your intended use is not permitted by statutory regulation or exceeds the permitted use, you will need to obtain permission directly from the copyright holder. To view a copy of this licence, visit http://creativecommons.org/licenses/by/4.0/. 


\section{References}

1. SAE International, Electric and Hybrid Electric Vehicle Rechargeable Energy Storage System (RESS) Safety and Abuse Testing (2009)

2. C.J. Orendorff, J. Lamb, L.A.M. Steele, Recommended Practices for Abuse Testing Rechargeable Energy Storage Systems (RESSs) (Sandia National Laboratories, Albuquerque, NM, 2017)

3. D.H. Doughty, C.C. Crafts, FreedomCAR Electrical Energy Storage System Abuse Test Manual for Electric and Hybrid Electric Vehicle Applications (Sandia National Laboratories, Albuquerque, NM, 2006)

4. Underwriters Laboratories, EESAs for On Road Vehicles and Off Road Industrial Vehicles, UL 2580 (2020)

5. Underwriters Laboratories, EESAs for Light Electric Vehicle (LEV) Applications, UL 2271 (2018)

6. J.A. Jeevarajan, C.S. Winchester, Battery safety qualifications for human ratings. Electrochem. Soc. Interface (2012)

7. J. Lamb, C.J. Orendorff, E.P. Roth, J. Langendorf, Studies on the thermal breakdown of common Li-ion battery electrolyte components. J. Electrochem. Soc. 162(10), A2131 (2015)

8. T.Y. Ma, S.Y. Wu, F. Wang, J. Lacap, C.J. Lin, S.Q. Liu, M.H. Wei, W.J. Hao, Y.S. Wang, J.W. Park, Degradation mechanism study and safety hazard analysis of overdischarge on commercialized lithium-ion batteries. ACS Appl. Mater. Interfaces 12(50), 56086 (2020)

9. M. Flugel, T. Waldmann, M. Kasper, M. Wohlfahrt-Mehrens, Detection of copper deposition on anodes of over-discharged lithium ion cells by GD-OES depth profiling. Chem. Phys. Chem. 21(18), 2047 (2020)

10. C.E. Hendricks, A.N. Mansour, D.A. Fuentevilla, G.H. Waller, J.K. Ko, M.G. Pecht Copper dissolution in overdischarged lithium-ion cells: $X$-ray photoelectron spectroscopy and $x$-ray absorption fine structure analysis. J. Electrochem. Soc. 167(9), 9 (2020)

11. D. Juarez-Robles, A.A. Vyas, C. Fear, J.A. Jeevarajan, P.P. Mukherjee, Overdischarge and aging analytics of Li-ion cells. J. Electrochem. Soc. 167(9), 15 (2020)

12. D. Juarez-Robles, J.A. Jeevarajan, P.P. Mukherjee, Degradation-safety analytics in lithium-ion cells: Part I Aging under charge/discharge cycling, J. Electrochem. Soc. 167(16), (2020)

13. Y. Preger, H.M. Barkholtz, A. Fresquez, D.L. Campbell, B.W. Juba, J. RomanKustas, S.R. Ferreira, B. Chalamala, Degradation of commercial lithium-ion cells as a function of chemistry and cycling conditions. J. Electrochem. Soc. 167(12), 9 (2020)

14. J.A. Jeevarajan, Safety of commercial lithium-ion cells and batteries, in LithiumIon Batteries-Advances and Applications, G. Pistoia, Ed. (Elsevier Ltd., Amsterdam, The Netherlands, 2014)

15. T. Joshi, S. Azam, C. Lopez, S. Kinyon, J. Jeevarajan, Safety of lithium-ion cells and batteries at different states-of-charge, J. Electrochem. Soc. 167(14), (2020)

16. J. Lamb, C.J. Orendorff, L.A.M. Steele, S.W. Spangler, Failure propagation in multi-cell lithium ion batteries. J. Power Sources 283, 517 (2015)

17. Test Method for Evaluating Thermal Runaway Fire Propagation in Battery Energy Storage Systems, UL 9540A (Underwriters Laboratories, Houston, 2016)

18. J. Straubel, D. Lyons, S.K. Berdichevsky, R. Teixeira, System and Method for Inhibiting the Propagation of an Exothermic Event (Tesla Inc.. Piscataway, NJ, 2007)

19. C.F. Lopez, J.A. Jeevarajan, P.P. Mukherjee, Characterization of lithium-ion battery thermal abuse behavior using experimental and computational analysis. $J$. Electrochem. Soc. 162(10), A2163 (2015)

20. E. Darcy, J. Darst, W. Walker, D. Finegan, P. Shearing, "Design Guidelines for Safe, High Performing Li-Ion Batteries With 18650 Cells," in JRC Exploratory Research Workshop (Joint Research Center, Petten, The Netherlands, 2018)

21. J.A. Jeevarajan, Hazards associated with high voltage high capacity lithium-ion batteries. ECS Trans. 33(22), 1 (2019)

22. R.M. Wittman, M.L. Perry, T.N. Lambert, B.R. Chalamala, Y. Preger, Perspective-on the need for reliability and safety studies of grid-scale aqueous batteries. J. Electrochem. Soc. 167(9), 8 (2020)

23. U. Koehler, "General Overview of Non-Lithium Battery Systems and their Safety Issues," Chapter 2, in Electrochemical Power Sources: Fundamentals, Systems, and Applications, J. Garche, K. Brandt, Eds. (Elsevier, Amsterdam, The Netherlands, 2019), pp. 21-46

24. B. Barnett, C. McCoy, D. Ofer, S. Sriramulu, Successful early detection of incipient internal short circuits in Li-ion batteries and prevention of thermal runaway. ECS Meet. Abstr. (2016)

25. N. Meddings, M. Heinrich, F. Overney, J.S. Lee, V. Ruiz, E. Napolitano, S. Seitz, G. Hinds, R. Raccichini, M. Gabers, J. Park, Application of electrochemical impedance spectroscopy to commercial Li-ion cells: A review. J. Power Sources 480, 27 (2020)

26. C.T. Love, M.B.V. Virji, R.E. Rocheleau, K.E. Swider-Lyons, State-of-health monitoring of $186504 \mathrm{~S}$ packs with a single-point impedance diagnostic. J. Power Sources 266, 512 (2014)

27. C.T. Love, K. Swider-Lyons, Impedance diagnostic for overcharged lithium-ion batteries. Electrochem. Solid-State Lett. 15(4), A53 (2012)

28. R. Srinivasan, A. CarsonBaisden, B.G. Carkhuff, M.H. Butler, The five modes of heat generation in a Li-ion cell under discharge. J. Power Sources 262, 93 (2014)
29. R. Srinivasan, B.G. Carkhuff, Empirical analysis of contributing factors to heating in lithium-ion cells: Anode entropy versus internal resistance. J. Power Sources 241, 560 (2013)

30. R. Srinivasan, B.G. Carkhuff, M.H. Butler, A.C. Baisden, Instantaneous measurement of the internal temperature in lithium-ion rechargeable cells. Electrochim. Acta 56(17), 6198 (2011)

31. J.L. Morrison, J.P. Christophersen, W.H. Morrison, "Universal Auto-Calibration for a Rapid Battery Impedance Spectrum Measurement Device," Presented at the IEEE Aerospace Conference, Big Sky, MT, March 1-18, 2014, p. 8

32. J.P. Christophersen, W.H. Morrison, J.L. Morrison, C.G. Motloch, D.M. Rose, "Crosstalk Compensation for a Rapid, Higher-Resolution Impedance Spectrum Measurement," Presented at the IEEE Aerospace Conference, Big Sky, MT, March 3-10, 2012, p. 16

33. J.P. Christophersen, D.F. Glenn, C.G. Motloch, R.B. Wright, C.D. Ho, V.S. Battaglia Electrochemical impedance spectroscopy testing on the advanced technology development program lithium-ion cells. IEEE Trans. Veh. Technol. 56(3), 1851 (2002)

34. J. Lamb, L. Torres-Castro, C. Orendorff, E. Dufek, L. Walker, C. Ho, Determination of Battery Stability with Advanced Diagnostics (Sandia National Laboratories, Albuquerque, NM, 2017)

35. S. Wilke, B. Schweitzer, S. Khateeb, S. Al-Hallaj, Preventing thermal runaway propagation in lithium ion battery packs using a phase change composite material: An experimental study. J. Power Sources 340, 51 (2017)

36. J.C. Hewson, H. Zhou, M. Parmananda, R.C. Shurtz, P.P. Mukherjee, From material properties to multiscale modeling to improve lithium-ion energy storage safety. MRS Bull. 46(5), 402 (2021)

37. D. Steingart, Thermo/Physical abuse couplings in batteries: From electrodes to cells. MRS Bull. 46(5), 410 (2021)

38. T. Tanim, E. Dufek, S.V. Sazhin, Challenges and needs for system-level electrochemical lithium-ion battery management and diagnostics. MRS Bull. 46(5), 420 (2021)

39. F. Takahashi, Fire blanket and intumescent coating materials for failure resistance. MRS Bull. 46(5), 429 (2021)

40. S. Sripad, A. Bills, V. Viswanathan, A review of safety considerations for batteries in aircraft with electric propulsion. MRS Bull. 46(5), 435 (2021)

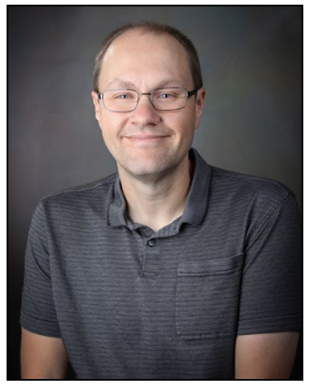

Joshua Lamb is currently a principal member of the Technical Staff with the Advanced Power Sources R\&D organization at Sandia National Laboratories. He is the lead for Battery Safety and Reliability for the US Department of Energy Office of Electricity energy-storage programs at Sandia. He also leads battery abuse testing at Sandia as part of the Battery Abuse Test Laboratory (BATLab) team. Lamb earned his PhD degree in metallurgical engineering in 2008 , and Lamb BS degree in chemical engineering in 2002 from the University of Nevada. His research interests include advanced techniques for determining the stability of lithium-ion batteries and the development of advanced battery abuse and safety tests. Lamb can be reached by email at jlamb@sandia.gov.

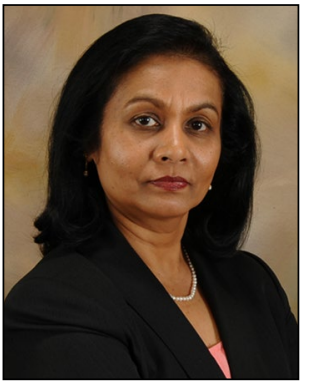

Judith A. Jeevarajan is a research director at Underwriters Laboratories. She serves in the Technical Working Group for standards organizations such as the International Electrotechnical Commission, Society of Automotive and Aerospace Engineers, International Civil Aviation Organization, American National Standards Institute, the United Nations (UN) Informal Working Group, and the American Institute of Aeronautics and Astronautics. She is a member of the Energy Advisory Council to the Great Lakes Energy Institute at Case Western Reserve University. Jeevarajan earned her PhD degree in chemistry (from The University of Alabama in 1995, and a MS degree in chemistry from the University of Notre Dame in 1991. She co-chairs a Battery Safety Council with the National Transportation Safety Board (NTSB). Her awards include the American Institute of Aeronautics and Astronautics Aerospace Power Systems Award, the India Energy Storage Alliance (IESA) as one of the leading women in the energy storage and electric vehicle industry, and the 2020 IESA Woman Leader of the Year Award in Energy Storage Systems. Jeevarajan can be reached by email at judy.jeevarajan@ul.org. 